\title{
Recovery after Acute Kidney Injury
}

\author{
John A. Kellum ${ }^{1,2}$, Florentina E. Sileanu ${ }^{1,2}$, Azra Bihorac ${ }^{3}$, Eric A. J. Hoste ${ }^{4}$, and Lakhmir S. Chawla ${ }^{5}$ \\ ${ }^{1}$ The Center for Critical Care Nephology, Department of Critical Care Medicine, University of Pittsburgh School of Medicine, Pittsburgh, \\ Pennsylvania; ' University of Pittsburgh Medical Center, Pittsburgh, Pennsylvania; ${ }^{3}$ Department of Anesthesiology, University of Florida, \\ Gainesville, Florida; ${ }^{4}$ Intensive Care Unit, Ghent University Hospital, Ghent, Belgium; and ${ }^{5}$ Department of Medicine, Veterans Affairs \\ Medical Center, Washington, DC
}

ORCID ID: 0000-0003-1995-2653 (J.A.K.).

\section{Abstract}

Rationale: Little is known about how acute kidney injury (AKI) resolves, and whether patterns of reversal of renal dysfunction differ among patients with respect to ultimate recovery.

Objectives: We sought to examine different patterns for AKI reversal that are found in patients and assess how they relate to postdischarge outcomes.

Methods: We studied 16,968 critically ill patients with Kidney Disease Improving Global Outcomes stage 2 or $3 \mathrm{AKI}$, using an electronic database. Reversal of AKI was defined as alive and no longer meeting criteria for even stage 1 . Recovery was defined as reversal at hospital discharge.

Measurements and Main Results: We observed five patterns. The most common $(4,508 ; 26.6 \%)$ was early reversal that was sustained through discharge, but almost as many patients $(4,496$;
$26.5 \%)$ had no reversal at all. The remaining patients had late reversal after Day 7 (9.7\%); early reversal with one or more relapses, but with ultimate recovery $(22.5 \%)$; and relapsing without recovery $(14.7 \%)$. Outcomes for patients with these phenotypes were quite different, with age-adjusted 1-year survival varying from more than $90 \%$ for early reversal to less than $40 \%$ for patients never reversing. Relapses are common (37.3\%), especially in the first 72 hours after reversal, and are associated with a fivefold increased risk for death by 1 year compared with early sustained reversal.

Conclusions: We have identified five distinct recovery phenotypes on the basis of the clinical course over the first week after AKI manifestation. These phenotypes may identify patients amenable to therapeutic intervention. Long-term outcomes are associated with recovery status at hospital discharge.

Keywords: critical care; outcomes; survival; dialysis; renal failure
More than a decade ago, an imperfect conceptual model for acute kidney injury (AKI) was proposed (1) and subsequently refined (2-4). Today, the consensus model serves as the basis for diagnosis, epidemiology, and clinical trials for AKI. The criteria specified in the model have been validated in terms of risk for death and dialysis $(5,6)$, have also served as the basis for biomarker discovery and validation
(7), and are in excellent agreement with expert adjudication (8). Although new research will inevitably further refine this conceptual model, there can be no doubt it has advanced the field in numerous ways.

A major limitation of the AKI model is that it is based on kidney function, not injury per se (9). As a result, it may follow injury, rather than identify it early. Although new biomarkers may improve early recognition $(7,8)$, they will not cause patients to seek medical attention earlier. This is compounded further by the fact that AKI does not have early symptoms that cause patients to seek care. Thus, for many forms of community-acquired AKI, we will likely need to accept that prevention will be impossible (10). Even for hospital-acquired AKI, prevention may be ineffective, and therefore our efforts will

(Received in original form April 18, 2016; accepted in final form September 16, 2016)

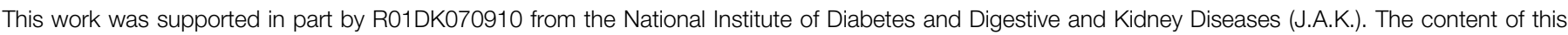

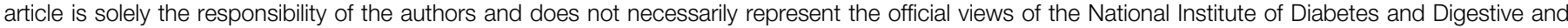
Kidney Diseases or the National Institutes of Health.

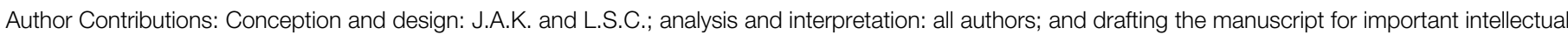
content: all authors.

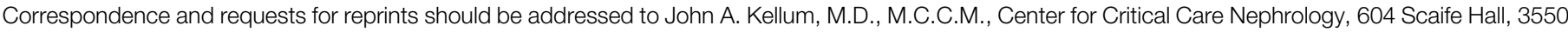
Terrace Street, Pittsburgh, PA 15261. E-mail: kellumja@upmc.edu

This article has an online supplement, which is accessible from this issue's table of contents at www.atsjournals.org

Am J Respir Crit Care Med Vol 195, Iss 6, pp 784-791, Mar 15, 2017

Copyright (C) 2017 by the American Thoracic Society

Originally Published in Press as DOI: 10.1164/rccm.201604-07990C on September 16, 2016

Internet address: www.atsjournals.org 


\section{At a Glance Commentary}

\section{Scientific Knowledge on the}

Subject: For many forms of acute kidney injury, prevention will not be possible because patients tend to present with acute kidney injury (AKI) that has already begun. Thus, efforts will need to target better recovery. Little is known about how recovery after AKI occurs or what different patterns of recovery mean for long-term outcomes.

\section{What This Study Adds to the}

Field: We have advanced a conceptual model of recovery from AKI, and we identified distinct recovery phenotypes on the basis of the clinical course over the first week. These phenotypes are associated with dramatically different outcomes.

need to target better recovery. Doing so will require a conceptual model, and may not be as simple as the reverse of the conceptual model for AKI. As we examine the various ways we might conceptualize recovery, it is clear that return of renal function may take on multiple trajectories $(11,12)$, and it is just as clear that these trajectories have not been described in any systematic way. For example, when renal function appears to normalize after AKI (we term this "reversal"), does this lead to recovery by hospital discharge? Are there differences between patients who have AKI reversal early in their course, as opposed to later? The various trajectories of renal recovery after AKI are also important because renal recovery is associated with long-term survival $(10,12,13)$ and has been identified as an important endpoint for clinical trials (14).

For reversal and recovery of AKI to be used as endpoints for clinical trials or to guide follow-up, or simply to be standardized so as to improve communication and research, there is a need for detailed analysis of patient data. Using this analysis, we hope to achieve a better understanding of how AKI reversal and, ultimately, renal recovery might be defined, and how various definitions might relate to long-term outcomes. Our proximate goals are to examine the different patterns for AKI reversal that are found in patients and assess how different time-based definitions for reversal describe these patterns. Our ultimate goal is to provide evidence for future development of a conceptual model for renal recovery that can be used both for trials and at the bedside.

\section{Methods}

\section{Conceptual Model}

For our analyses, we developed a working conceptual model for the study of the kinetics of AKI reversal and renal recovery (Figure 1) based on prior literature (1-6, 11). Because AKI is an abrupt loss of renal function developing over 7 days or less (1), and because the actual start of AKI may not be identified, we defined "early reversal" as absence of AKI for at least a 24-hour period within 7 days of the first documented onset of AKI. We operationalized this conceptual model by limiting our analysis to patients with stage 2 or $3 \mathrm{AKI}$, and defined reversal as the absence of any AKI stage 1-3. This was done to avoid misclassification of minor changes in renal function (e.g., just above and below the cutoffs for staging) or influences of fluid balance. We determined final recovery status at hospital discharge, recognizing that recovery may occur after discharge in some patients. To examine the effect of the duration of AKI reversal, we applied several rules. If multiple episodes of reversal occurred (i.e., reversal followed by relapse of stage 2 or $3 \mathrm{AKI}$ ), we considered the longest instance that reversal was sustained. We considered death before

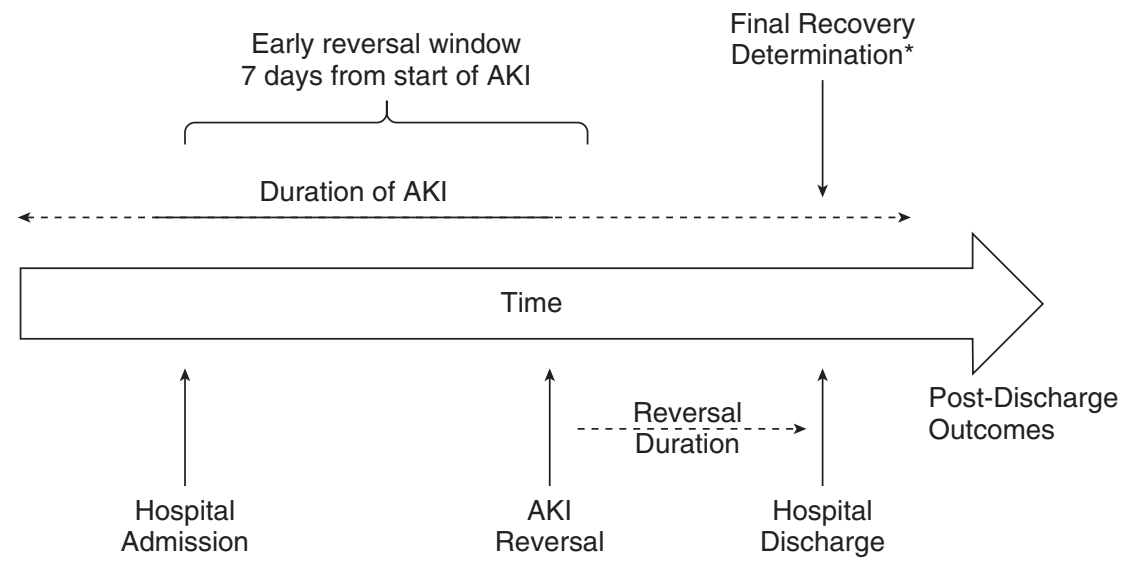

Figure 1. Conceptual model. Acute kidney injury (AKI) is an abrupt loss of renal function developing over the course of 7 days or less (1). Although the actual start of AKI may not be identified (more than half of AKI cases are community acquired), and many cases may not resolve rapidly, we defined "early reversal" as absence of AKI for at least 24 hours within 7 days of first documented AKI. ${ }^{*}$ Recovery status was determined at hospital discharge, but we recognize that recovery may proceed out further, and a true final status would be better assessed at 90 days or longer. hospital discharge as "nonrecovery" because renal recovery without survival is both rare and not patient-centered. This analysis provides three separate pieces of information. First, we can identify five groups of patients: those with AKI reversal within the first 7 days of reaching stage 2 or 3 that was sustained through hospital discharge, those without AKI reversal within the first 7 days of reaching stage 2 or 3 who may or may not be sustained through hospital discharge, and those with a "stuttering course" with at least one relapse that may or may not resolve before hospital discharge. Second, we can observe how these various groups differ in terms of outcomes at hospital discharge and out to 1 year. Third, we can better understand the patients exhibiting AKI reversal by stratifying on the basis of the duration of reversal. This is important because patients are treated in prospect, and we should understand the risks for relapse as a function of how long after the initial reversal such relapses occur. Said another way: How long should a patient be followed closely after their kidney dysfunction has resolved? We can also determine sensitivity and specificity for different duration of AKI reversal for predicting recovery at hospital discharge.

\section{Patients}

Approval was obtained from the University of Pittsburgh Institutional Review Board. We analyzed data on 45,568 adult patients admitted to any of 
eight intensive care units (ICUs) at the University of Pittsburgh Medical Center during an 8-year period (July 2000-October 2008). After excluding patients who never developed stage 2 or 3 AKI or received hemodialysis or renal transplant before hospital admission, those with known baseline creatinine $\geqslant 3.5 \mathrm{mg} / \mathrm{dl}$, those receiving large blood transfusion ( $>4$ units packed red blood cells within any 24-hour period), or those with incomplete data (see Figure 2), our analysis cohort included 16,968 patients with stage 2 and 3 AKI.

\section{Variables and Outcomes}

We calculated the acute physiology components of the Acute Physiology and Chronic Health Evaluation III (APACHE III) score (APS-III) (15), and defined suspected sepsis as the ordering of blood cultures and antibiotics within 24 hours of each other, as defined previously (16). Baseline, admission, and reference serum creatinine were determined as previously described $(5,17,18)$. We classified patients according to the KDIGO (Kidney Disease Improving Global Outcomes) criteria (4), using serum creatinine (SC) and urine output (UO) criteria. Hourly SC was interpolated linearly from point to point, provided that the gap between 2 recorded values was $\leqslant 48$ hours. If two SC values were separated by more than 48 hours, the second value became the new starting point for the interpolation. The last available SC value was dragged down to the next integer hour. Available UO data were redistributed hourly, as previously described (5). If multiple episodes of AKI occurred, we only considered the first occurrence of stage 2 or 3 AKI for purposes of defining reversal. Subsequent events were considered "relapses."

We defined AKI reversal as the absence of any stage of AKI by either SC or UO criteria. For example, a patient with stage $2 \mathrm{AKI}$ would have to have a decrease in SC to less than $150 \%$ of baseline, and be free of periods of oliguria ( $\mathrm{UO}<5 \mathrm{ml} / \mathrm{kg} / \mathrm{hr}$ ) longer than 6 hours. For each patient, we determined the timing and duration of reversal. We ascertained AKI status in 24-hour intervals from the time of reversal during the first 7 days from stage 2 or 3 AKI.

Any subsequent occurrence of stage 2 or 3 AKI after initial reversal was considered a relapse. After 7 days, we only assessed AKI status again at hospital discharge. To be defined as completely recovered at

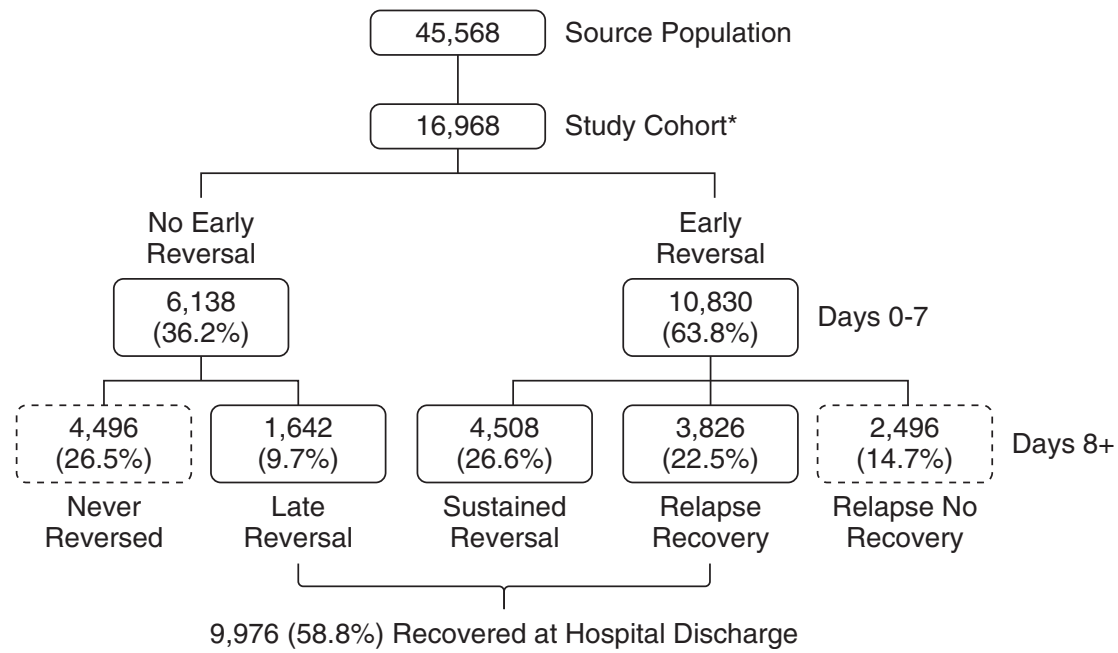

Figure 2. Study flow. Source population included all patients admitted to the intensive care unit (ICU). *Exclusions: not stage 2 or 3 acute kidney injury (23,587); end-stage kidney disease, creatinine $>3.5$ or receiving dialysis before ICU admission (3,839); massive blood transfusion $(1,174)$; ICU stay $<48$ hours $(3,631)$.

discharge, a patient had to be alive, and in the 3 calendar days before discharge, off renal replacement therapy and free of $\mathrm{AKI}$ by either SC or UO criteria. Thus, a total of five patterns of recovery can be identified: early sustained reversal (defined as reversal starting within 7 days and sustained through hospital discharge), late reversal (defined as reversal after 7 days and sustained through hospital discharge), relapsing AKI with complete recovery at hospital discharge, relapsing AKI without complete recovery at hospital discharge, and never-reversed AKI. We considered death before hospital discharge as nonrecovery because renal recovery without survival is both rare and not patient-centered, as suggested by international consensus recommendations $(11,14)$.

For patients who exhibited AKI reversal within the 7-day "early reversal window," we calculated the duration of reversal in days. If there were multiple reversal periods, we only considered the longest reversal period for purposes of duration. For convenience, and to limit the total number of groups to those representing $>10 \%$, we considered all durations $\geqslant 5$ days $(120 \mathrm{~h})$ as one group. For patients discharged before 5 days after first meeting reversal criteria, we assigned these patients to the $\geqslant 5$-day group if their reversal was sustained through discharge. In the unusual event that a patient died while meeting reversal criteria, the duration of reversal was truncated to the time of death.
Our primary outcomes were survival and need for chronic dialysis at 1 year after ICU admission. Vital status was determined from the National Center for Health Statistics National Death Index database or the Social Security Administration's Death Master File. Chronic dialysis was determined by querying the U.S. Renal Data System database.

\section{Statistical Analysis}

Statistical analyses were performed using the STATA software, version SE 13.1 (StataCorp, College Station, TX), with statistical significance set at $P<0.05$. Survival graphs were created using the R package "survival," version 2.38-1 (https://www.r-project.org). Comparisons across groups were performed using the $\chi^{2}$ asymptotic test for categorical variables, and the Kruskal-Wallis one-way analysis of variance by ranks for continuous variables. We used multivariable logistic regression to identify risk factors associated with recovery at hospital discharge, late reversal versus no reversal of AKI. Wald tests were used to assess the significance of each risk factor retained in the final models reporting individual odds ratios (ORs) for each. Cox proportional hazards model was used to graphically describe the age-adjusted survival at 1 year after ICU admission. In the survival model, the Breslow method was used for ties, the likelihood ratio test was used to test the overall statistical significance of the model, and Wald 
tests were used to do pairwise comparisons between groups.

\section{Results}

\section{Patients and Recovery Status at Discharge}

Among the 16,968 patients with KDIGO stage 2 or 3 AKI (Figure 2) 9,976 (58.8\%) patients had complete recovery of renal function (no longer met any criteria for AKI) at hospital discharge. Patients recovering at hospital discharge can be further subdivided into those with early sustained reversal $(4,508 ; 26.6 \%)$, those with late sustained reversal (1,642; 9.7\%), and those with relapsing AKI who ultimately recovered by discharge $(3,826$; $22.5 \%$ ). The remaining 6,992 patients who did not have complete renal recovery at hospital discharge can also be subdivided into those who exhibited reversal, but then relapsed without subsequent recovery $(2,496 ; 14.7 \%)$, and those who had no AKI reversal at any point $(4,496 ; 26.5 \%)$. Baseline characteristics for these patients are provided in Table 1. Patient characteristics associated with nonrecovery in multivariable models are shown in Tables 2-4. Table 2 shows a model with the outcome of nonrecovery at hospital discharge. Increasing age and comorbidities increased the odds of not recovering, whereas surgical admission and increasing urine output favored recovery. Table 3 examines the variables associated with recovery in patients who do not exhibit early reversal (i.e., comparing late reversal with no reversal). Older patients, blacks, medical patients, patients with lower reference creatinine, and those without respiratory failure all had higher odds of not recovering (Table 3 ). Table 4 compares patients with a relapse (with or without subsequent recovery) with patients with early sustained reversal. Interestingly, sepsis is a strong indicator of a relapse (OR, 1.34; 95\% confidence interval [CI], 1.18-1.52; $P<0.001$ ), as is black race (OR, $1.37 ; 95 \%$ CI, 1.13-1.67; $P<0.001$ ).

\section{AKI Trajectories}

Figure 2 illustrates three patterns of patient course based on evidence for AKI reversal in the first week after stage 2 or 3 criteria were met. The most common pattern observed $(37.3 \%)$ was a relapsing course in which patients met reversal criteria but did not remain without AKI on subsequent days. The second most common pattern was nonreversal in the first week (36.2\%). The prognoses for relapsing versus nonrecovery in the first week were quite different, with $60.5 \%$ of the former group $(3,826$ of 6,322$)$ ultimately recovering renal

Table 1. Patient Characteristics Stratified by Patterns of Acute Kidney Injury Reversal

\begin{tabular}{|c|c|c|c|c|c|c|}
\hline Characteristic* & $\begin{array}{c}\text { Early Sustained } \\
\text { Reversal } \\
(N=4,508)\end{array}$ & $\begin{array}{l}\text { Late Sustained } \\
\text { Reversal } \\
(N=1,642)\end{array}$ & $\begin{array}{l}\text { Relapse, } \\
\text { Recovery } \\
(N=3,826)\end{array}$ & $\begin{array}{c}\text { Relapse, No } \\
\text { Recovery } \\
(N=2,496)\end{array}$ & $\begin{array}{c}\text { Never } \\
\text { Reversed } \\
(N=4,496)\end{array}$ & $\boldsymbol{P}$ Value $^{\dagger}$ \\
\hline Age, yr, median (Q1-Q3) & $60(49-72)$ & $64(52-75)$ & $65(53-76)$ & $67(54-77)$ & $67(54-77)$ & $<0.001$ \\
\hline Males & $2,630(58.3)$ & $924(56.3)$ & $2,226(58.2)$ & $1,374(55)$ & $2,381(53)$ & $<0.001$ \\
\hline Race & & & & & & $<0.001$ \\
\hline White & $3,618(80.3)$ & $1,321(80.5)$ & $3,021(79)$ & $1,932(77.4)$ & $3,348(74.5)$ & \\
\hline Black & $355(7.9)$ & $129(7.9)$ & $291(7.6)$ & $169(6.8)$ & $323(7.2)$ & \\
\hline Other & 535 (11.9) & $192(11.7)$ & $514(13.4)$ & $395(15.8)$ & 825 (18.3) & \\
\hline BMI, kg/m², median (Q1-Q3) & $28.1(24.4-33.0)$ & $28.8(24.7-34.9)$ & $27.7(24.1-32.3)$ & $27.4(23.6-32.2)$ & $27.4(23.6-32.9)$ & $<0.001$ \\
\hline \multicolumn{7}{|l|}{ Comorbid conditions } \\
\hline Hypertension & $1,514(33.6)$ & $597(36.4)$ & $1,438(37.6)$ & $938(37.6)$ & $1,619(36)$ & 0.001 \\
\hline Diabetes & 787 (17.5) & $353(21.5)$ & $816(21.3)$ & $550(22)$ & 927 (20.6) & $<0.001$ \\
\hline Cardiac disease & 745 (16.5) & $351(21.4)$ & $820(21.4)$ & 565 (22.6) & 997 (22.2) & $<0.001$ \\
\hline Liver transplant & $118(2.6)$ & $54(3.3)$ & $103(2.7)$ & $70(2.8)$ & $149(3.3)$ & 0.24 \\
\hline Chronic renal disease & $191(4.2)$ & $105(6.4)$ & $191(5)$ & $131(5.2)$ & $278(6.2)$ & $<0.001$ \\
\hline Multiple comorbidities & 2,097 (46.5) & 797 (48.5) & 1,988 (52) & $1,286(51.5)$ & $2,252(50.1)$ & $<0.001$ \\
\hline Surgical admission & $2,857(68.5)$ & $1,102(70.2)$ & $2,568(70.8)$ & $1,548(66.2)$ & $2,176(51.9)$ & $<0.001$ \\
\hline \multicolumn{7}{|l|}{$\begin{array}{l}\text { Creatinine, mg/dl, } \\
\text { median (Q1-Q3) }\end{array}$} \\
\hline Hospital admission & $1(0.8-1.3)$ & $1.1(0.9-1.8)$ & $1(0.8-1.4)$ & $1(0.8-1.4)$ & $1.4(0.9-2.3)$ & $<0.001$ \\
\hline Known baseline value & $0.9(0.7-1.2)$ & $1(0.7-1.3)$ & $0.9(0.7-1.2)$ & $0.9(0.7-1.2)$ & $1(0.7-1.3)$ & 0.001 \\
\hline Reference value & $0.9(0.8-1.1)$ & $0.9(0.8-1.1)$ & $0.9(0.7-1.1)$ & $0.9(0.7-1)$ & $0.9(0.8-1.1)$ & $<0.001$ \\
\hline $\begin{array}{l}\text { APS-III score }{ }^{\ddagger} \\
\text { median (Q1-Q3) }\end{array}$ & $59(43-79)$ & $63(45-84)$ & $66(50-85)$ & 70 (52-90) & $75(54-101)$ & $<0.001$ \\
\hline $\begin{array}{l}\text { Weight-adjusted urine } \\
\text { output, ml/kg, } \\
\text { median (Q1-Q3) }\end{array}$ & $17.9(12.9-26.8)$ & $15.8(10.2-24.8)$ & $17.5(12.5-26.5)$ & $17.4(11.8-26.4)$ & $14.2(7.3-23.9)$ & $<0.001$ \\
\hline Suspected sepsis & $520(11.5)$ & $223(13.6)$ & $593(15.5)$ & $414(16.6)$ & $883(19.6)$ & $<0.001$ \\
\hline Vasopressors ${ }^{\ddagger}$ & 989 (21.9) & $511(31.1)$ & $981(25.6)$ & 749 (30) & $1,845(41)$ & $<0.001$ \\
\hline Mechanical ventilation ${ }^{\ddagger}$ & 2,665 (59.1) & $1,059(64.5)$ & $2,484(64.9)$ & 1,656 (66.3) & $2,792(62.1)$ & $<0.001$ \\
\hline
\end{tabular}

Definition of abbreviations: APS-III = Acute Physiology and Chronic Health Evaluation III; BMI = body mass index; ICU = intensive care unit; $Q=$ quartile. Data are shown as $n(\%)$ unless otherwise indicated.

*Missing data: age $(n=4)$, BMI $(n=3,313)$, surgical admission $(n=1,073)$, hospital admission creatinine $(n=1,071)$, known baseline value $(n=10,015)$, APS-III score $(n=22)$, weight-adjusted urine output $(n=670)$.

${ }^{\dagger}$ Pearson chi-square asymptotic test for categorical variables; Kruskal-Wallis test for continuous variables.

${ }^{\ddagger}$ Captured in the 24 hours after ICU admission.

§The available urine in the 24 hours after ICU admission was summed and divided by the weight. 
Table 2. Multivariable Logistic Regression Models for Renal Recovery Outcome: No Recovery versus Recovery

\begin{tabular}{lccc}
\hline Variable & OR & 95\% Cl for OR & $P$ Value \\
Age, by 5 yr & & & \\
Male & 1.04 & $1.03-1.06$ & $<0.001$ \\
Race & 0.87 & $0.82-0.94$ & $<0.001$ \\
$\quad$ Black vs. white & 0.93 & $0.81-1.06$ & 0.26 \\
$\quad$ Other vs. white & 1.28 & $1.16-1.42$ & $<0.001$ \\
Hypertension & 0.89 & $0.82-0.97$ & 0.005 \\
Cardiac disease $_{\text {Surgical admission }}$ & 1.14 & $1.04-1.25$ & 0.007 \\
APS-III score*, by units of 10 & 0.64 & $0.59-0.68$ & $<0.001$ \\
Weight-adjusted urine output $^{\dagger}$, by $100 \mathrm{ml}$ & 1.13 & $1.11-1.14$ & $<0.001$ \\
Vasopressors $^{*}$ & 0.54 & $0.43-0.68$ & $<0.001$ \\
Mechanical ventilation $^{*}$ & 1.51 & $1.40-1.62$ & $<0.001$ \\
\hline
\end{tabular}

Definition of abbreviations: APS-III = Acute Physiology and Chronic Health Evaluation III;

$\mathrm{Cl}=$ confidence interval; $\mathrm{ICU}=$ intensive care unit; $\mathrm{OR}=$ odds ratio.

$\mathrm{N}=15,266$; area under the receiver operating characteristic curve $(95 \% \mathrm{Cl})=0.647(0.638-0.656)$; goodness-of-fit $P$ value $=0.37$.

${ }^{*}$ Captured in the 24 hours after ICU admission.

tThe available urine volume in the 24 hours after ICU admission was summed and divided by the weight.

function, and only $26.8 \%(1,642$ of 6,138$)$ of patients with no reversal from $0-7$ days ultimately recovering $(P<0.001)$. For patients who exhibited early sustained reversal, the median time to reversal was 30 hours versus 43 hours $(P<0.001)$ and 47 hours $(P<$ $0.001)$ for patients with relapse either with subsequent recovery or not, respectively.

\section{Outcomes}

Unadjusted outcomes by recovery status are shown in Table 5. Early reversal was associated with the shortest ICU and hospital lengths of stay and best prognosis (1-yr survival, 90.2\%), and those patients who never recovered or had reversal with relapse had the longest lengths of stay and worst prognosis (1-yr survival, about $40 \%$ ). Patients recovering late did better than those with no recovery, but not as well as those recovering early (AKI reversal sustained). Oneyear age-adjusted survival by group is shown in Figure 3. The same patterns observed in unadjusted analyses were seen in the adjusted model, in that early reversal does best, but late reversal still does better than no reversal.

\section{Duration of AKI Reversal}

Patients exhibiting AKI reversal within the early reversal window are further stratified by the duration of reversal in Table E1 in the online supplement. When we examine the

Table 3. Multivariable Logistic Regression Models for Renal Recovery Outcome: No Reversal versus Late Reversal

\begin{tabular}{lccr}
\hline Variable & OR & 95\% Cl for OR & P Value \\
Age, by 5 yr & 1.02 & $1.00-1.04$ & 0.03 \\
Race & 1.42 & $1.07-1.89$ & 0.01 \\
$\quad$ Black vs. white & 1.09 & $0.86-1.38$ & 0.48 \\
$\quad$ Other vs. white & 0.54 & $0.47-0.62$ & $<0.001$ \\
Surgical admission & 0.85 & $0.76-0.94$ & 0.002 \\
Reference creatinine* & 1.14 & $1.11-1.17$ & $<0.001$ \\
APS-III score*, by 10 units $_{\text {Vasopressors* }}$ & 1.40 & $1.22-1.60$ & $<0.001$ \\
Mechanical ventilation $^{*}$ & 0.75 & $0.64-0.86$ & $<0.001$ \\
\hline
\end{tabular}

Definition of abbreviations: APS-III = Acute Physiology and Chronic Health Evaluation III; $\mathrm{Cl}=$ confidence interval; $\mathrm{ICU}=$ intensive care unit; $\mathrm{OR}=$ odds ratio.

$\mathrm{N}=5,405$; area under the receiver operating characteristic curve $(95 \% \mathrm{Cl})=0.661(0.646-0.678)$; goodness-of-fit $P$ value $=0.79$.

${ }^{*}$ Captured in the 24 hours after ICU admission. course for patients exhibiting an AKI reversal event within the first week after meeting stage 2 or 3 criteria, we can see that 10,830 patients $(63.8 \%)$ had reversal of renal dysfunction at least temporarily. For 6,077 patients $(35.8 \%)$ this reversal was sustained for at least 5 consecutive days (sum of the last two columns). An additional 840 patients $(5 \%)$ had reversal for 4 days, and 969 (5.7\%) had reversal lasting for 3 days. Finally, we found an additional 1,347 (7.9\%) and 1,597 (9.4\%) patients who had AKI reversal that was sustained for only 48 or 24 hours, respectively. Thus, depending on how sustained the AKI reversal (i.e., duration), the percentage of patients classified as AKI reversal varied from $63.8 \%(1 \mathrm{~d})$ to $35.8 \%$ (5 d). Characteristics for these various patient groups are shown in Table E1. Finally, if we ask how well various durations of reversal predict recovery at hospital discharge, we can see sensitivity, specificity, and positive and negative predictive values in Table E2. Specificity for recovery at hospital discharge does not exceed $80 \%$ until reversal is sustained for at least 72 hours.

\section{Sensitivity Analyses}

To help assess generalizability, we included treatment year as a variable in our models, as practice patterns may change (as do providers) over time. Our results remained unchanged. Finally, we repeated our primary analysis after changing the definition of reversal/recovery to be less stringent (considered to be reached when no AKI stage 2 or 3 was present; i.e., stage 1 was considered reversal/recovery). Our results are summarized in Table E3 and are virtually identical to our primary analysis (Tables 1 and 5). We also repeated the models shown in Tables 2-4 after removing in-hospital deaths. The results were similar (Table E4).

\section{Discussion}

To our knowledge, this is the first study to characterize the various patterns of recovery after an episode of AKI and relate these patterns to long-term outcomes. Our results have implications for patient care and healthcare policy. For example, nonrecovery after AKI is common (approximately 40\%) and associated with rather poor prognosis. Indeed, patients who were not recovered at hospital discharge (whether they had early reversal or not) were twice as likely to be dead at 1 year (59\%) compared with 
Table 4. Multivariable Logistic Regression Models for Renal Recovery Outcome: Relapse versus Early Sustained Reversal

\begin{tabular}{lccc}
\hline Variable & OR & 95\% Cl for OR & $P$ Value \\
Age, by 5 yr & 1.05 & $1.03-1.06$ & $<0.001$ \\
Race & & & $<0.001$ \\
$\quad$ Black vs. white & 1.37 & $1.13-1.67$ & 0.001 \\
$\quad$ Other vs. white & 1.01 & $0.86-1.18$ & 0.9 \\
Diabetes & 1.16 & $1.04-1.29$ & 0.01 \\
Cardiac disease & 1.30 & $1.16-1.46$ & $<0.001$ \\
APS-III score*, by 10 units & 1.08 & $1.06-1.1$ & $<0.001$ \\
Suspected sepsis* $^{\star}$ & 1.34 & $1.18-1.52$ & 0.001 \\
Mechanical ventilation* $^{*}$ & 1.13 & $1.03-1.24$ & 0.01 \\
\hline
\end{tabular}

Definition of abbreviations: APS-III = Acute Physiology and Chronic Health Evaluation III;

$\mathrm{Cl}=$ confidence interval; $\mathrm{ICU}=$ intensive care unit; $\mathrm{OR}=$ odds ratio.

$\mathrm{N}=9,861$; area under the receiver operating characteristic curve $(95 \% \mathrm{Cl})=0.661(0.6-0.622)$;

goodness-of-fit $P$ value $<0.001$

${ }^{*}$ Captured in the 24 hours after ICU admission.

patients who experienced late recovery (with or without early reversal; $29 \%$ ).

Interestingly, late recovery is also very common with more than half of patients who ultimately recover, doing so after day 7 (i.e., no early reversal or relapsing).

Although these patients do not achieve the same survival statistics as patients with early sustained reversal, their prognosis is markedly better than those who do not recover, and these differences persist for at least 1 year after discharge. Given the frequency and effect on survival for late recovery, efforts to understand this phenomenon and target it for therapies would seem critical. Indeed, early reversal usually occurred within the first 72 hours (Table E1), and fully half of all early reversal occurred in the first 30 hours, perhaps before many treatments could be initiated. Focusing on identification of patients who have not had early reversal could dramatically change the current treatment paradigm.

Another interesting result of our analysis is that recovery that appears to start (early reversal), but then relapse or recur is actually the most common pattern after AKI. This unstable pattern has a better prognosis than patients not exhibiting any improvement within the first week: $60.5 \%$ ultimately recovering renal function compared with only $26.8 \%$. However, there may be opportunities to stabilize these patients (effectively moving them into the early recovery group) or increase their numbers by moving patients out of the nonrecovery group. Unfortunately, modifiable risk factors were not identified (Tables 2 and 3), but various at-risk groups can be seen. One such group is defined by lower baseline serum creatinine. Worse outcomes from AKI in patients with lower creatinine has been observed previously (19), and could reflect poor surveillance or general health deficits (decreased muscle mass).

Finally, as we follow patients clinically who exhibit recovery after AKI, we need to keep in mind that specificity for predicting recovery at discharge is rather low until about 72 hours. Patients with 24 or even 48 hours of reversal still commonly "slip back." Thus, careful monitoring of these patients would seem to be indicated. In addition, this observation may have value on deciding when it is safe to reinitiate medications that may have been discontinued during the initiation of AKI. Given the results of late recovery, it likely just as important not to "write off" kidney function in a patient who has no evidence of early reversal.

Our study does have important limitations. Although we studied a large number of patients from several ICUs, our patients were all cared for in a single large healthcare system with practice patterns that may be different from other institutions. Mitigating this concern somewhat is our observation that over time (as providers and treatment practices change), our results remain stable. Furthermore, our overall rates of AKI and outcomes agree well with a recent international cross-sectional study performed in 97 centers (6), and our results indicating low mortality for patients with recovery are consistent with our prior work in patients with septic shock from a 31-center trial of resuscitation strategies (10). Our decision to study patients with

Table 5. Outcomes Stratified by Recovery Pattern

\begin{tabular}{|c|c|c|c|c|c|c|}
\hline Outcome & $\begin{array}{c}\text { Early Sustained } \\
\text { Reversal }(N=4,508)\end{array}$ & $\begin{array}{c}\text { Late Sustained } \\
\text { Reversal }(N=1,642)\end{array}$ & $\begin{array}{c}\text { Relapse, Recovery } \\
\text { (N=3,826) }\end{array}$ & $\begin{array}{c}\text { Relapse, No } \\
\text { Recovery }(N=2,496)\end{array}$ & $\begin{array}{c}\text { Never Reversed } \\
(N=4,496)\end{array}$ & $P$ Value* \\
\hline In-hospital RRT, n (\%) & $\mathrm{n} / \mathrm{a}$ & $248(15.1)$ & $75(2)$ & $264(10.6)$ & $964(21.4)$ & $<0.001$ \\
\hline \multicolumn{7}{|l|}{$\begin{array}{l}\text { Length of stay, d, } \\
\text { median (Q1-Q3) }\end{array}$} \\
\hline ICU & $4(3-7)$ & $6(3-14)$ & $7(4-15)$ & $8(4-16)$ & $5(3-10)$ & $<0.001$ \\
\hline Hospital & $12(8-18)$ & $17(9-32)$ & $20(11-31)$ & $23(14-38)$ & $12(6-24)$ & $<0.001$ \\
\hline \multicolumn{7}{|l|}{ Mortality, n (\%) } \\
\hline ICU & $\mathrm{n} / \mathrm{a}$ & $n / a$ & $\mathrm{n} / \mathrm{a}$ & 545 (21.8) & 1,559 (34.7) & $<0.001$ \\
\hline Hospital & $\mathrm{n} / \mathrm{a}$ & $\mathrm{n} / \mathrm{a}$ & $\mathrm{n} / \mathrm{a}$ & $1,141(45.7)$ & $2,154(47.9)$ & 0.08 \\
\hline $30 \mathrm{~d}$ & $44(1)$ & $43(2.6)$ & $124(3.2)$ & 889 (35.6) & $2,034(45.2)$ & $<0.001$ \\
\hline $90 \mathrm{~d}$ & $159(3.5)$ & 173 (10.5) & 519 (13.6) & $1,246(49.9)$ & $2,400(53.4)$ & $<0.001$ \\
\hline $365 d$ & $443(9.8)$ & $414(25.2)$ & 1,177 (30.8) & $1,450(58.1)$ & 2,687 (59.8) & $<0.001$ \\
\hline
\end{tabular}

Definition of abbreviations: ICU = intensive care unit; $\mathrm{n} / \mathrm{a}=$ not applicable; $\mathrm{Q}=$ quartile; $\mathrm{RRT}=$ renal replacement therapy.

Where there is an $\mathrm{n} / \mathrm{a}$, the $P$ value represents the comparison between groups with values.

*Pearson chi-square asymptotic test for categorical variables; Kruskal-Wallis test for continuous variables. 


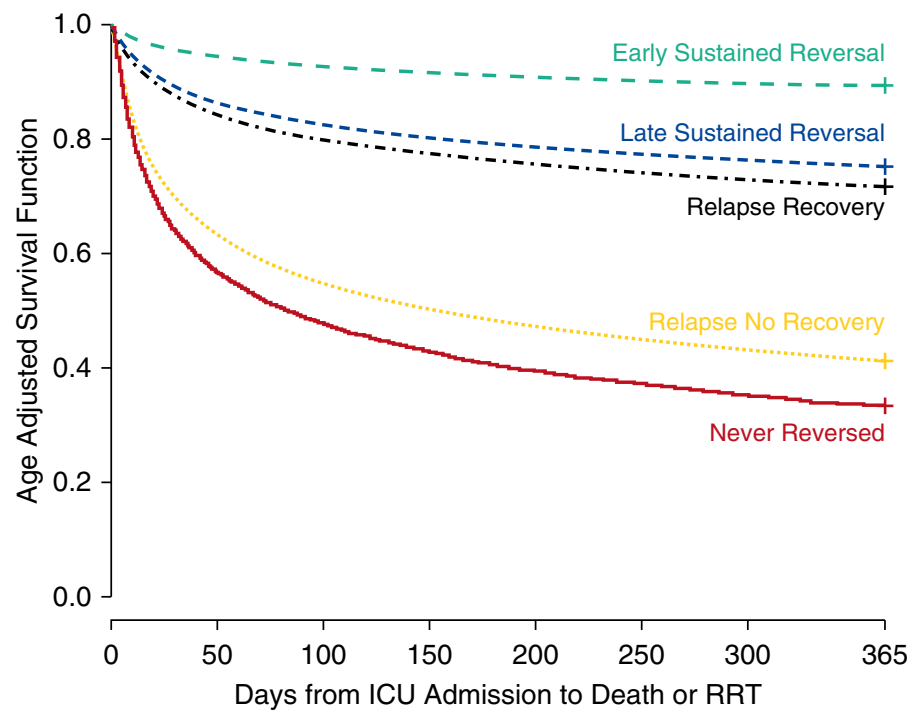

No. at risk

$\begin{array}{lllllllll}\text { Early Sustained Reversal } & 4,507 & 4,404 & 4,317 & 4,235 & 4,176 & 4,122 & 4,070 & 4,026 \\ \text { Late Sustained Reversal } & 1,642 & 1,529 & 1,424 & 1,357 & 1,310 & 1,272 & 1,242 & 1,203 \\ \text { Relapse Recovery } & 3,823 & 3,535 & 3,245 & 3,061 & 2,910 & 2,818 & 2,719 & 2,625 \\ \text { Relapse No Recovery } & 2,496 & 1,386 & 1,190 & 1,114 & 1,075 & 1,052 & 1,027 & 1,008 \\ \text { Never Reversed } & 4,496 & 2,127 & 1,922 & 1,826 & 1,757 & 1,709 & 1,678 & 1,648\end{array}$

Figure 3. Age-adjusted survival by recovery patterns. Survival differences are highly significant overall $(P<0.001)$. All pairwise comparisons are also significant. For example, late sustained reversal versus relapse recovery hazard ratio (95\% confidence interval): $0.86(0.77-0.96), P=0.005$; relapse with no recovery versus never reversed hazard ratio (95\% confidence interval): 0.81 (0.76-0.86), $P<0.001$. ICU = intensive care unit; RRT = renal replacement therapy.

moderate to severe AKI (stage 2 or 3 ) was pragmatic because distinguishing clinically relevant AKI from mild fluctuations in renal function can be quite challenging for stage 1. Similar pragmatism led us to exclude patients with short ICU stays or receiving large-volume blood transfusions, as missing or uninterpretable data (creatinine) could be an issue in such patients. Finally, the definitions for renal recovery have not been standardized, and although consensus exists regarding basic principles $(1,20)$, variation in specific implementations abound (11). Our primary definition, absence of "any AKI," has construct validity, but may be no better than other definitions. In particular, we cannot exclude ongoing "subclinical" injury. However, return of function is a fairly a common index for recovery in other areas of medicine. Future studies might expand recovery to include dynamic measures of function that could be more sensitive $(21,22)$. The results of our various sensitivity analyses are reassuring in that our primary results were robust to changing our definitions to treat return to stage 1 or less as reversal/recovery.

In conclusion, we have identified at least three distinct recovery phenotypes on the basis of the clinical course over the first week after AKI manifestation (early reversal, relapsing, and no reversal). These phenotypes are associated with dramatically different long-term outcomes. However, long term outcomes are associated with recovery status at hospital discharge. We have proposed a conceptual model for AKI recovery to guide future research.

Author disclosures are available with the text of this article at www.atsjournals.org.

\section{References}

1. Bellomo R, Ronco C, Kellum JA, Mehta RL, Palevsky P; Acute Dialysis Quality Initiative workgroup. Acute renal failure - definition, outcome measures, animal models, fluid therapy and information technology needs: the Second International Consensus Conference of the Acute Dialysis Quality Initiative (ADQI) Group. Crit Care 2004;8:R204-R212.

2. Mehta RL, Kellum JA, Shah SV, Molitoris BA, Ronco C, Warnock DG, Levin A; Acute Kidney Injury Network. Acute Kidney Injury Network: report of an initiative to improve outcomes in acute kidney injury. Crit Care 2007;11:R31.

3. Akcan-Arikan A, Zappitelli M, Loftis LL, Washburn KK, Jefferson LS, Goldstein SL. Modified RIFLE criteria in critically ill children with acute kidney injury. Kidney Int 2007;71:1028-1035.

4. Kidney Disease: Improving Global Outcomes (KDIGO) Acute Kidney Injury Work Group. KDIGO clinical practice guideline for acute kidney injury. Kidney Int Suppl 2012;2:1-138.

5. Kellum JA, Sileanu FE, Murugan R, Lucko N, Shaw AD, Clermont G. Classifying AKI by Urine Output versus Serum Creatinine Level. J Am Soc Nephrol 2015;26:2231-2238.

6. Hoste EAJ, Bagshaw SM, Bellomo R, Cely CM, Colman R, Cruz DN, Edipidis $\mathrm{K}$, Forni LG, Gomersall CD, Govil D, et al. Epidemiology of acute kidney injury in critically ill patients: the multinational AKI-EPI study. Intensive Care Med 2015;41:1411-1423.
7. Kashani K, Al-Khafaji A, Ardiles T, Artigas A, Bagshaw SM, Bell M, Bihorac A, Birkhahn R, Cely CM, Chawla LS, et al. Discovery and validation of cell cycle arrest biomarkers in human acute kidney injury. Crit Care 2013;17:R25.

8. Bihorac A, Chawla LS, Shaw AD, Al-Khafaji A, Davison DL, Demuth GE, Fitzgerald R, Gong MN, Graham DD, Gunnerson K, et al. Validation of cell-cycle arrest biomarkers for acute kidney injury using clinical adjudication. Am J Respir Crit Care Med 2014;189:932-939.

9. Murray PT, Mehta RL, Shaw A, Ronco C, Endre Z, Kellum JA, Chawla LS, Cruz D, Ince C, Okusa MD; ADQI 10 workgroup. Potential use of biomarkers in acute kidney injury: report and summary of recommendations from the 10th Acute Dialysis Quality Initiative consensus conference. Kidney Int 2014;85:513-521.

10. Kellum JA, Chawla LS, Keener C, Singbartl K, Palevsky PM, Pike FL, Yealy DM, Huang DT, Angus DC. ProCESS and ProGReSS-AKI investigators: the effects of alternative resuscitation strategies on acute kidney injury in patients with septic shock. Am J Respir Crit Care Med 2016;193:281-287.

11. Kellum JA. How can we define recovery after acute kidney injury? Considerations from epidemiology and clinical trial design. Nephron Clin Pract 2014;127:81-88.

12. Korenkevych D, Ozrazgat-Baslanti T, Thottakkara P, Hobson CE, Pardalos P, Momcilovic P, Bihorac A. The pattern of longitudinal change in serum creatinine and 90 -day mortality after major surgery. Ann Surg 2016;263:1219-1227. 
13. Bihorac A, Yavas S, Subbiah S, Hobson CE, Schold JD, Gabrielli A, Layon AJ, Segal MS. Long-term risk of mortality and acute kidney injury during hospitalization after major surgery. Ann Surg 2009;249: 851-858.

14. Palevsky PM, Molitoris BA, Okusa MD, Levin A, Waikar SS, Wald R, Chertow GM, Murray PT, Parikh CR, Shaw AD, et al. Design of clinical trials in acute kidney injury: report from an NIDDK workshop on trial methodology. Clin J Am Soc Nephrol 2012;7: 844-850.

15. Knaus WA, Wagner DP, Draper EA, Zimmerman JE, Bergner M, Bastos PG, Sirio CA, Murphy DJ, Lotring T, Damiano A, et al. The APACHE III prognostic system. Risk prediction of hospital mortality for critically ill hospitalized adults. Chest 1991;100:1619-1636.

16. Dellinger RP, Levy MM, Rhodes A, Annane D, Gerlach H, Opal SM, Sevransky JE, Sprung CL, Douglas IS, Jaeschke R, et al.; Surviving Sepsis Campaign Guidelines Committee including the Pediatric Subgroup. Surviving sepsis campaign: international guidelines for management of severe sepsis and septic shock: 2012. Crit Care Med 2013;41:580-637.
17. Hoste EAJ, Clermont G, Kersten A, Venkataraman R, Angus DC, De Bacquer D, Kellum JA. RIFLE criteria for acute kidney injury are associated with hospital mortality in critically ill patients: a cohort analysis. Crit Care 2006;10:R73.

18. Závada J, Hoste E, Cartin-Ceba R, Calzavacca P, Gajic O, Clermont G, Bellomo R, Kellum JA; AKI6 investigators. A comparison of three methods to estimate baseline creatinine for RIFLE classification. Nephrol Dial Transplant 2010;25:3911-3918.

19. Mehta RL, Pascual MT, Gruta CG, Zhuang S, Chertow GM. Refining predictive models in critically ill patients with acute renal failure. J Am Soc Nephrol 2002;13:1350-1357.

20. Goldstein SL, Chawla L, Ronco C, Kellum JA. Renal recovery. Crit Care 2014;18:301.

21. Chawla LS, Davison DL, Brasha-Mitchell E, Koyner JL, Arthur JM, Shaw AD, Tumlin JA, Trevino SA, Kimmel PL, Seneff MG. Development and standardization of a furosemide stress test to predict the severity of acute kidney injury. Crit Care 2013;17:R207.

22. Fuhrman DY, Kellum JA. Biomarkers for diagnosis, prognosis and intervention in acute kidney injury. Contrib Nephrol 2016;187:47-54. 